\title{
Editorial Statistics and Best Reviewers Award for 2014
}

\author{
Hiroaki Shimokawa, MD, $\mathrm{PhD}$
}

\section{Dear Colleagues,}

On behalf of the Circulation Journal, I am pleased to announce the editorial statistics for 2014, and the recipients of the Best Reviewers Awards for 2014.

\section{Editorial statistics for 2014}

As shown in Figure 1, we received 1,402 manuscripts in 2014, the highest number on record, for publication in the Journal, including 1,060 original papers (910 clinical and 150 experimental), 58 invited review articles, 100 invited editorials, 31 rapid communications, and 130 images in cardiovascular medicine. Approximately half were submitted from Japan, followed by China, Korea, the U.S.A., Taiwan and Italy (Figure 2). For the last 12 years, the number of submissions has been steadily increasing even after we terminated publication of case reports in October 2008 and the East Japan Earthquake Disaster (Figure 3). The abovementioned 6 countries have remained
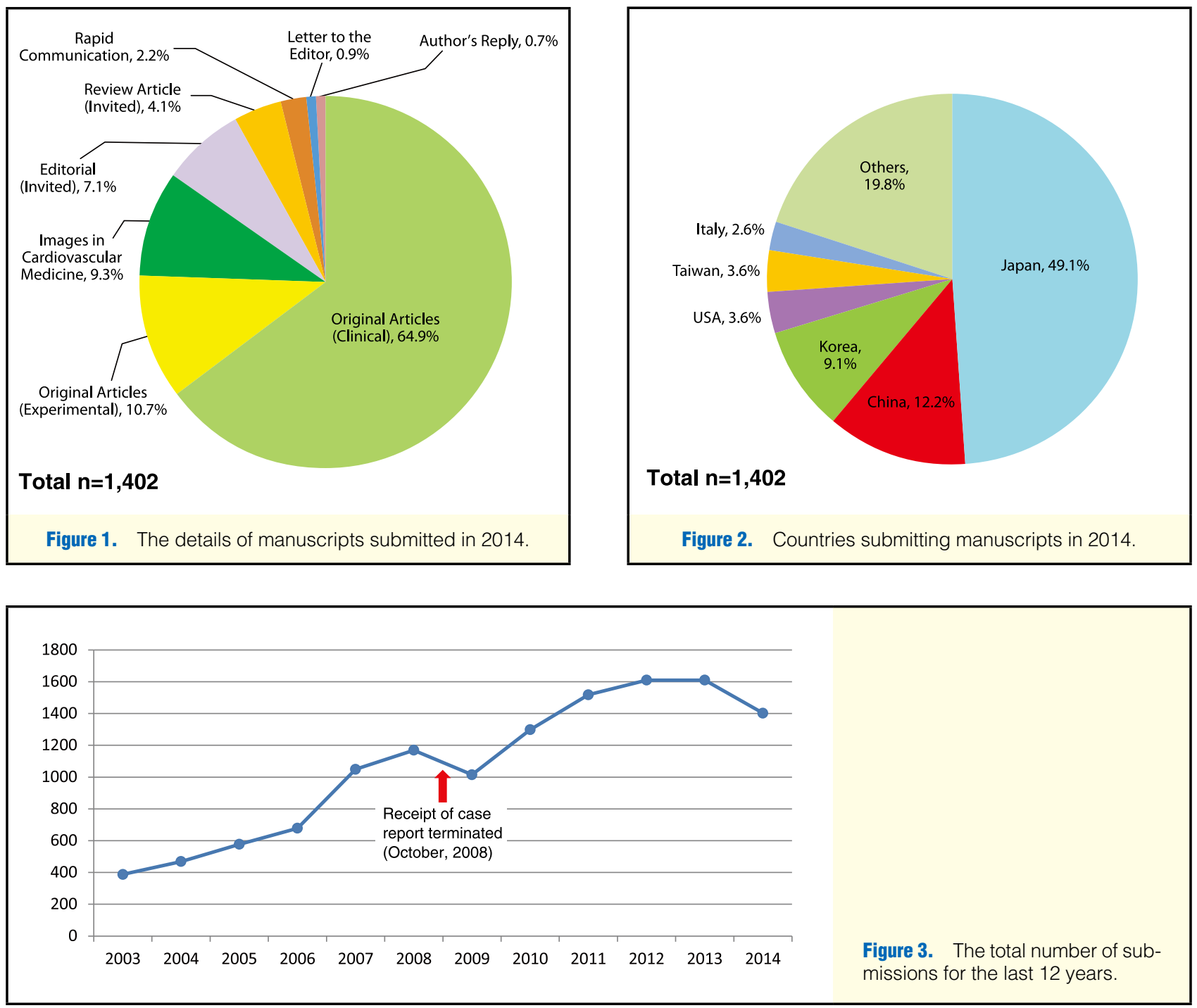


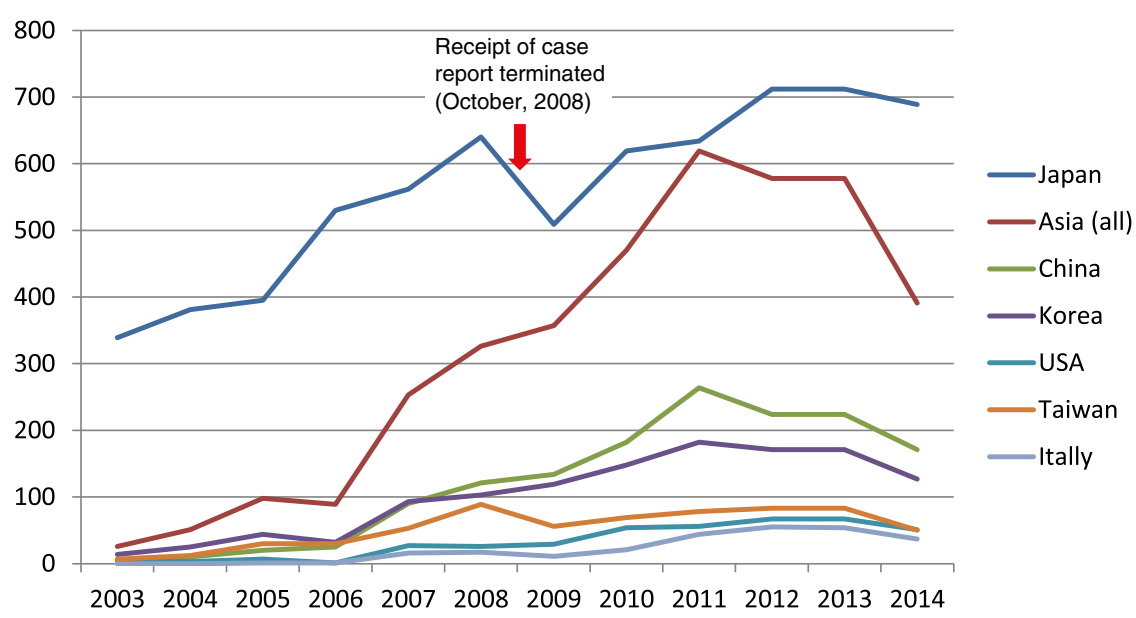

Figure 4. The number of submissions for the last 12 years in top 6 countries. Asia (All): all Asian countries except Japan.

the top 6 countries in terms of paper submissions over that period (Figure 4). The acceptance rate for original manuscripts in 2014 was approximately $22.9 \%$.

\section{Best Reviewers Awards for 2014}

In 2014, a total of 814 reviewers kindly reviewed the manuscripts submitted. In appreciation of their valuable contributions, we have selected the 20 Best Reviewers of the Year 2014 as follows:
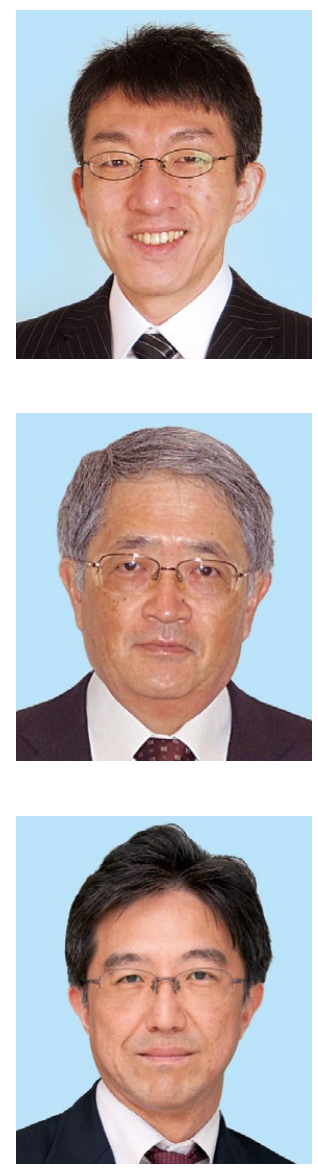

\section{Hiroshi Asanuma, MD}

(Kyoto Prefectural University of Medicine, Kyoto)

Number of reviews: 24

RPS: 134.0

Previous awards: 2009, 2010, 2011, 2012, 2013

\section{Akira Fujiki, MD}

(Shizuoka Red Cross Hospital, Shizuoka)

Number of reviews: 24

RPS: 122.9

Previous awards: 2009, 2010, 2011, 2012, 2013

\section{Koichiro Kinugawa, MD}

(The University of Tokyo, Tokyo)

Number of reviews: 21

RPS: 114.0

Previous awards: 2013

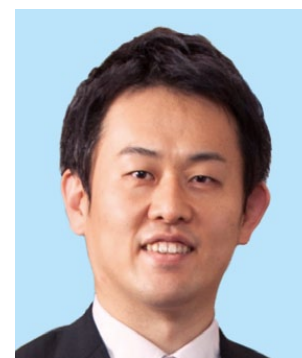

Kyung-Duk Min, MD

(National Cerebral and Cardiovascular Center, Osaka)

Number of reviews: 23

RPS: 125.0

Previous awards: 2013

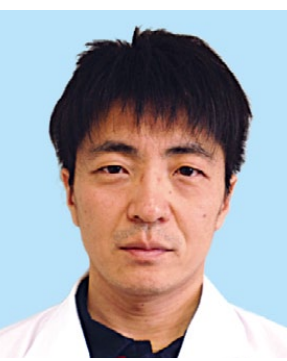

Osamu Tsukamoto, MD

(Osaka University Graduate

School of Medicine, Osaka)

Number of reviews: 22

RPS: 119.8

Previous awards: 2012, 2013

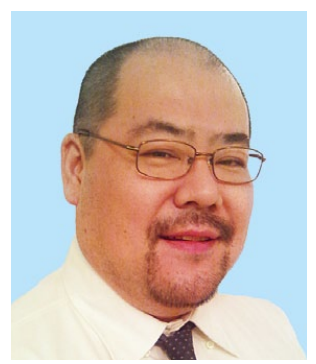

\section{Shoji Sanada, MD}

(Ministry of Health, Labour and Welfare, Government of Japan, Tokyo)

Number of reviews: 17

RPS: 89.6

Previous awards: 2008,2009 , 2010, 2011, 2012, 2013 


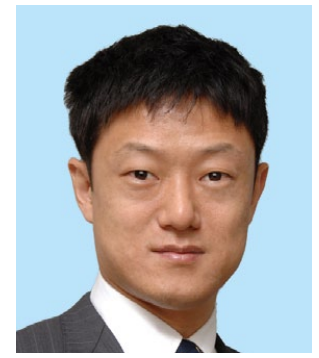

Kenichi Fujii, MD

(Hyogo College of Medicine, Hyogo)

Number of reviews: 15

RPS: 87.8

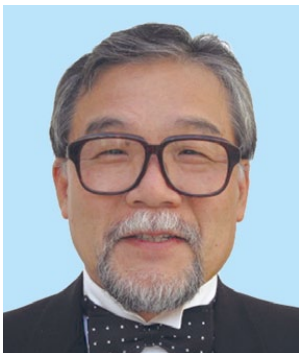

\section{Yutaka Okita, MD}

(Kobe University, Hyogo)

Number of reviews: 14

RPS: 77.5

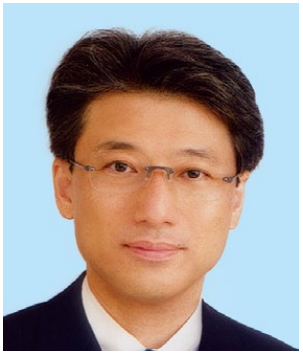

Masaharu Ishihara, MD

(Hyogo College of Medicine, Hyogo)

Number of reviews: 15

RPS: 71.5

Previous awards: 2009, 2010,

2011, 2013

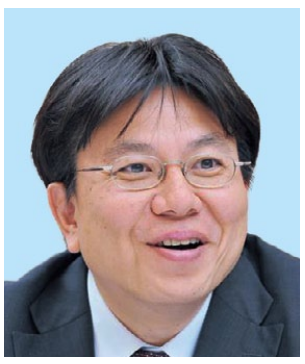

Kengo Fukushima Kusano, MD

(National Cerebral and

Cardiovascular Center, Osaka)

Number of reviews: 13

RPS: 69.1

Previous awards: 2012

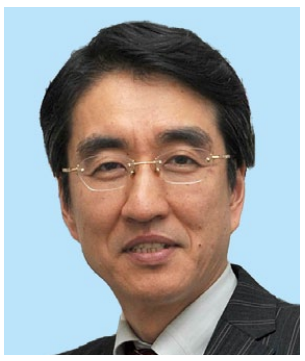

Takanori Ikeda, MD

(Toho University Faculty of

Medicine, Tokyo)

Number of reviews: 14

RPS: 65.0

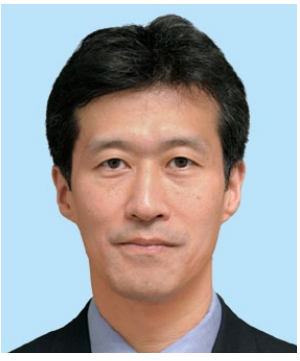

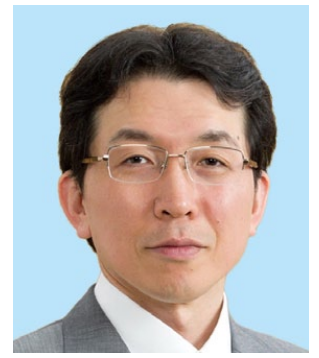

Kazuhiro Yamamoto, MD

(Tottori University, Tottori)

Number of reviews: 13

RPS: 82.8

Previous awards: 2008, 2010, 2011, 2012, 2013

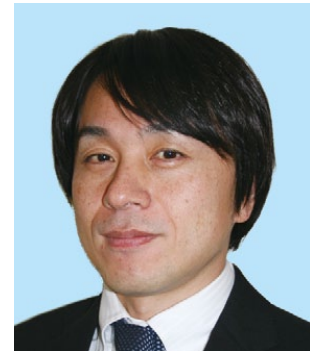

\section{Takashi Koyama, MD}

(Akita University, Akita)

Number of reviews: 14

RPS: 76.8

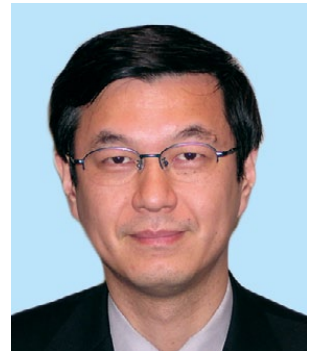

\section{Shinichi Niwano, MD}

(Kitasato University School of Medicine, Kanagawa)

Number of reviews: 11

RPS: 69.7

Previous awards: 2009, 2010, 2011, 2012, 2013

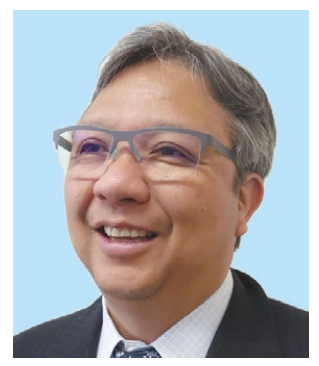

Masamichi Hirose, MD

(Iwate Medical University, Iwate)

Number of reviews: 12 RPS: 67.2

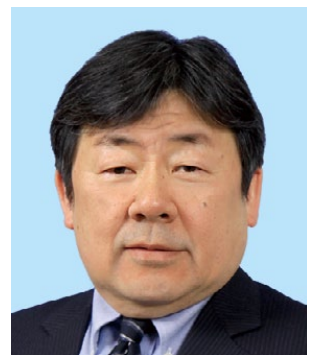

Akihiko Nogami, MD

(University of Tsukuba, Ibaraki)

Number of reviews: 11 RPS: 62.9

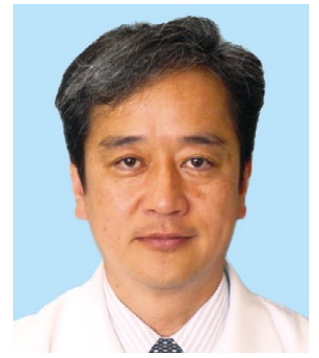

\section{Naohiko Takahashi, MD}

(Oita University, Oita)

Number of reviews: 12

RPS: 60.0

Previous awards: 2011, 2012 , 


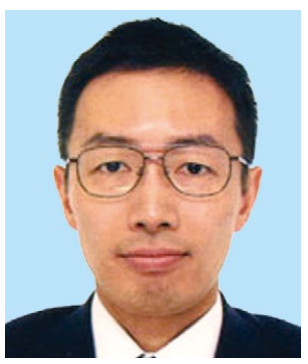

Hiroyuki Morita, MD

(The University of Tokyo, Tokyo)

Number of reviews: 10

RPS: 60.0

Previous awards: 2013

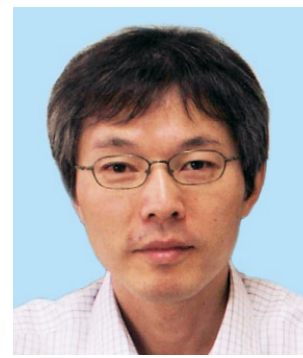

Tetsushi Furukawa, MD

(Tokyo Medical and Dental

University, Tokyo)

Number of reviews: 11

RPS: 59.5

Previous awards: 2009, 2011,

2012, 2013

*The Reviewer Performance Score (RPS) is determined as follows for reviewers who made at least 6 reviews in 2014 (review time limit, 14 days):

*RPS $=[($ Number of reviews completed within 1 week $) \times 2+($ number of reviews completed within 2 weeks $) \times 1+($ number of reviews completed within 3 weeks $) \times 0.2] \times$ quality of review (average) - (number of declined reviews $)+($ number of editorial comments published in the Journal $) \times 10$.

The review quality of reviewers was evaluated for each manuscript by an Associate Editor who was in charge of the manuscript (range: 1 3) and the average score used.

The above Reviewers will be awarded by the Japanese Circulation Society with a Certificate of Achievement and an honorarium.

In the year ahead in 2015, we expect a steady increase in the number of submissions, as well as an increase in the scientific impact of the Journal.

Hiroaki Shimokawa, MD, PhD

Editor-in-Chief

Circulation Journal

(Released online January 16, 2015) 